九州大学学術情報リポジトリ

Kyushu University Institutional Repository

\title{
On the $\mathrm{SO}(\mathrm{N})$ and $\mathrm{Sp}(\mathrm{N})$ free energy of a rational homology three-sphere
}

Takata, Toshie

Department of Mathematics, Faculty of Mathematics, Kyushu University

http://hdl. handle. net/2324/25493

出版情報: International Journal of Mathematics. 22 (4)，pp.465-482，2011-04. World Scientific Publishing Company

バージョン:

権利関係: (C) 2012 World Scientific Publishing Company 


\title{
ON THE $S O(N)$ AND $S p(N)$ FREE ENERGY OF A RATIONAL HOMOLOGY 3-SPHERE
}

\author{
TOSHIE TAKATA
}

\section{Dedicated to Professor Akio Kawauchi on the occasion of his 60th birthday}

Faculty of Mathematics, Kyushu University, Fukuoka, JAPAN

ttakata@math.kyushu-u.ac.jp

\begin{abstract}
We give an explicit presentation of the $S O(N)$ and $S p(N)$ free energy of lens spaces and show that the genus $g$ terms of it are analytic in a neighborhood at zero, where we can choose the neighborhood independently of $g$. Moreover, we prove that for any rational homology 3-sphere $M$ and any $g$, the genus $g$ terms of $S O(N)$ and $S p(N)$ free energy of $M$ agree up to sign. We also observe new weight systems related to the free energy.
\end{abstract}

\section{INTRODUCTION}

Let $G_{N}$ be a compact Lie group parameterized by $N$ such as $S U(N), S O(N)$ or $S p(N)$, and let $\mathfrak{g}_{N}$ be the Lie algebra of $G_{N}$. The LMO invariant $Z_{M} \in \mathcal{A}(\emptyset)$ [6] of a closed 3-manifold $M$ is presented by a linear sum of (a kind of) trivalent graphs, where $\mathcal{A}(\emptyset)$ denotes the $\mathbb{Q}$ vector space spanned by such trivalent graphs (subject to some relations). The $\mathfrak{g}_{N}$ weight system $W_{\mathfrak{g}_{N}}$ is a map $\mathcal{A}(\emptyset) \rightarrow \mathbb{Q}[[h]]$ which "substitutes" $\mathfrak{g}_{N}$ to trivalent graphs, such that $W_{\mathfrak{g}_{N}}(D)$ of a trivalent graph $D$ of degree $d$ is defined to be $h^{d}$ times some polynomial in $N$ of degree $\leq d+2$. When we fix a value of $N, W_{\mathfrak{g}_{N}}\left(\log Z_{M}\right)$ is a power series in $h$ with $\mathbb{Q}$ coefficients, which presents the perturbative expansion of the path integral of the Chern-Simons theory on the trivial $G_{N}$ bundle over $M$. When we regard $N$ as a variable, the weight system can be regarded as a map $W_{\mathfrak{g}_{\star}}: \mathcal{A}(\emptyset) \rightarrow \mathbb{Q}[N][[h]]$, and $W_{\mathfrak{g}_{\star}}\left(\log Z_{M}\right)$ is a power series in $h$ whose coefficients are polynomials in $N$. Putting $\tau$ to be $N h$ if $G_{N}=S U(N),(N-1) h$ if $G_{N}=S O(N)$, and $(N+1) h$ if $G_{N}=S p(N), W_{\mathfrak{g}_{\star}}\left(\log Z_{M}\right)$ is a power series in $\tau$ and $h$. We denote it by $F_{M}^{G_{N}}(\tau, h) \in h^{-2} \mathbb{Q}[[\tau, h]]$, and call it the $G_{N}$ free energy of $M$ [5]. Further, we put the coefficient of $h^{g-2}$ in $F_{M}^{G_{N}}(\tau, h)$ to be $F_{M, g}^{G_{N}}(\tau) \in \mathbb{Q}[[\tau]]$, i.e.,

$$
F_{M}^{G_{N}}(\tau, h)=\sum_{g=0}^{\infty} h^{g-2} F_{M, g}^{G_{N}}(\tau),
$$

where the value of $g$ implies the genus of some surface appearing in the definition of the weight system.

Recently, in [4], S. Garoufalidis, T.T.Q. Le and M. Mariño proved that the power series $F_{M, g}^{S U(N)}(\tau)$ of a closed oriented 3-manifold $M$ for any $g$ is analytic in a neighborhood of zero, where the neighborhood is independent of $g$, and gave an explicit presentation of the $S U(N)$ free energy for lens spaces to illustrate the analyticity. Further, S. Sinha and C. Vafa [8] gave a formula of the $S O(N)$ and $S p(N)$ free energy of $S^{3}$ from Chern-Simons gauge theory. 
In this paper, when $G_{N}=S O(N)$ and $S p(N)$, we give an explicit presentation of the $G_{N}$ free energy for lens spaces, and show that $F_{L(d, b), g}^{G_{N}}(\tau)$ of the lens space $L(d, b)$ is analytic in a neighborhood of zero, where we can choose the neighborhood independently of $g$ (Theorem 2). This analyticity has been conjectured by Le, Garoufalidis and Mariño [4]. Moreover, we show that for any $g$, the genus $g$ terms of $S O(N)$ and $S p(N)$ free energy agree up to sign (Corollary 2 ) and observe new weight systems related to the $G_{N}$ free energy (Section 4).

An idea of the proof of Theorem 2 is to use a presentation of $F_{L(d, b), g}^{G_{N}}(\tau)$ given in [4], which is a presentation in terms of the sum of some function of $h$ over positive roots of $\mathfrak{g}_{N}$. We calculate this sum concretely when $G_{N}=S O(N)$ and $S p(N)$, to present $F_{L(d, b), g}^{G_{N}}(\tau)$ by a function of $\tau$ and $h$.

The paper is organized as follows. In Section 2, we review the definition of the $G_{N}$ free energy and results on the $S U(N)$ free energy for lens spaces obtained by Garoufalidis, Le and Mariño. In Section 3, we present an explicit presentation of the $S O(N)$ and $S p(N)$ free energy for lens spaces and study these analyticity. We also show that the genus $g$ terms of $S O(N)$ and $S p(N)$ free energy for a rational homology 3-sphere agree up to sign. In Section 4, we recall properties of the $\mathfrak{s l}_{N}$ and $\mathfrak{s o}_{N}$ weight systems and observe new weight systems related to the free energy. In Section 5, we prove a relation between the $\mathfrak{s o}_{N}$ and $\mathfrak{s p}_{N}$ weight systems.

Acknowledgment The author wishes to thank S. Garoufalidis, T. Ohtsuki, D. Bar-Natan, T.T.Q. Le, and M. Mariño for valuable comments.

\section{Preliminaries}

In this section, we review the definition of the free energy and some results about the $S U(N)$ free energy of lens spaces in [4].

We briefly review the LMO invariant $Z_{M}$ of a closed oriented 3-manifold $M$, constructed by T.T.Q. Le, J. Murakami and T. Ohtsuki in [6]. We denote by $\mathcal{A}(\emptyset)$ the vector space over $\mathbb{Q}$ spanned by trivalent graphs whose vertices are oriented, modulo the AS, IHX and STU relations and denote by $\mathcal{A}(\emptyset)_{\text {conn }}$ the subspace of $\mathcal{A}(\emptyset)$ spanned by connected trivalent graphs. The degree of a trivalent graph is half the number of vertices. The LMO invariant $Z_{M}$ takes values in $\mathcal{A}(\emptyset)$. It is known that $\log Z_{M}$ takes values in $\mathcal{A}(\emptyset)_{\text {conn }}$.

Let us recall the weight system associated with a semi-simple Lie algebra $\mathfrak{g}$. It is known that for a semi-simple Lie algebra $\mathfrak{g}$, one obtains a $\mathbb{Q}$ linear map $W_{\mathfrak{g}}: \mathcal{A}(\emptyset) \rightarrow \mathbb{Q}[[h]]$, called the weight system associated with $\mathfrak{g}$ (for general references, see $[2,7]$ ). From a trivalent graph $D$ of degree $d$ in $\mathcal{A}(\emptyset), W_{\mathfrak{g}}(D)$ is obtained by substituting $\mathfrak{g}$ into $D$, contracting a tensor at vertices and multiplying by $h^{d}$. When $\mathfrak{g}=\mathfrak{g}_{N}=\mathfrak{s l}_{N}, \mathfrak{s o}_{N}$ or $\mathfrak{s p}_{N}$, regarding $N$ as a variable, $W_{\mathfrak{g}_{N}}(D)$ of a connected trivalent graph $D$ of degree $d$ is $h^{d}$ times some polynomial in $N$ of degree $\leq d+2$ by Lemma 1 below, and we regard the weight system $W_{\mathfrak{g}_{N}}$ as a map $W_{\mathfrak{g}_{\star}}: \mathcal{A}(\emptyset) \rightarrow \mathbb{Q}[N][[h]]$.

Lemma 1. For $\mathfrak{g}_{N}=\mathfrak{s l}_{N}, \mathfrak{s o}_{N}, \mathfrak{s p}_{N}$ and a connected trivalent graph $D$ of degree $d, W_{\mathfrak{g}_{N}}(D)$ can be presented in the following form,

$$
W_{\mathfrak{g}_{N}}(D)=\sum_{0 \leq g \leq d+1} a_{\mathfrak{g}_{N}, g}(D) N^{d+2-g} h^{d}
$$

for some $a_{\mathfrak{g}_{N}, g}(D) \in \mathbb{Z}$.

We show a proof of the lemma in Section 4.

Let $G_{N}$ be a simple compact Lie group $S U(N), S O(N)$ or $S p(N)$ and let $\mathfrak{g}_{N}$ be the Lie algebra of $G_{N}$. Putting $\tau$ to be $N h$ for $\mathfrak{g}=\mathfrak{s l},(N-1) h$ for $\mathfrak{g}=\mathfrak{s o}$, and $(N+1) h$ for $\mathfrak{g}=\mathfrak{s p}$, 
$W_{\mathfrak{g}_{\star}}(D)$ has the following form,

$$
W_{\mathfrak{g}_{\star}}(D)=\sum_{0 \leq g \leq d+1} c_{\mathfrak{g}, g}(D) \tau^{d+2-g} h^{g-2},
$$

for some $c_{\mathfrak{g}, g}(D) \in \mathbb{Z}$. Since $\log Z_{M} \in A(\emptyset)_{\text {conn }}, W_{\mathfrak{g}_{\star}}\left(\log Z_{M}\right)$ can be presented in the following form,

$$
W_{\mathfrak{g}_{\star}}\left(\log Z_{M}\right)=\sum_{d>0} \sum_{0 \leq g \leq d+1} c_{\mathfrak{g}, d, g}(M) \tau^{d+2-g} h^{g-2} \in h^{-2} \mathbb{Q}[[\tau, h]],
$$

for some $c_{\mathfrak{g}, d, g}(M) \in \mathbb{Q}$. As in [4], we define the $G_{N}$ free energy of a rational homology 3-sphere $M$ by

$$
F_{M}^{G_{N}}(\tau, h):=W_{\mathfrak{g}_{\star}}\left(\log Z_{M}\right) \in h^{-2} \mathbb{Q}[[\tau, h]],
$$

and put the coefficient of $h^{g-2}$ in $F_{M}^{G_{N}}(\tau, h)$ to be $F_{M, g}^{G_{N}}(\tau) \in \mathbb{Q}[[\tau]]$, i.e.,

$$
F_{M}^{G_{N}}(\tau, h)=\sum_{g=0}^{\infty} F_{M, g}^{G_{N}}(\tau) h^{g-2} .
$$

Let us review a presentation of the $G_{N}$ free energy of a lens space given in [4]. Let $L(d, b)$ be the lens space of type $(d, b)$. It is shown in [4] that, for any semi-simple Lie algebra $\mathfrak{g}$,

$$
W_{\mathfrak{g}_{\star}}\left(Z_{L(d, b)}\right)=\exp \left(\frac{\lambda_{L(d, b)}}{4} C_{\mathfrak{g}} \cdot \operatorname{dim} \mathfrak{g} \cdot h\right) d^{\left|\Psi_{+}\right|} \prod_{\alpha \in \Psi_{+}} \frac{\sinh ((\alpha, \rho) h /(2 d))}{\sinh ((\alpha, \rho) h / 2)},
$$

where $\lambda_{M}$ denotes Casson-Walker invariant for a rational homology 3-sphere $M, \Psi_{+}$denotes the set of positive roots of $\mathfrak{g},\left|\Psi_{+}\right|$denotes the number of positive roots, and $C_{\mathfrak{g}}$ is the quadratic Casimir of $\mathfrak{g}$. Since $F_{L(d, b)}^{G_{N}}(\tau, h)=W_{\mathfrak{g}_{\star}}\left(\log Z_{L(d, b)}\right)=\log W_{\mathfrak{g}_{\star}}\left(Z_{L(d, b)}\right)$ by definition, we obtain the following proposition from (4).

Proposition 2 ([4, Proposition 6.1]).

$$
F_{L(d, b)}^{G_{N}}(\tau, h)=\frac{\lambda_{L(d, b)}}{4} C_{\mathfrak{g}_{N}} \cdot \operatorname{dim} \mathfrak{g}_{N} \cdot h+\sum_{\alpha \in \Psi_{+}}(f((\alpha, \rho) h / d)-f((\alpha, \rho) h)),
$$

where we define the function $f$ by

$$
f(x):=\log \left(\frac{\sinh (x / 2)}{x / 2}\right) .
$$

By a concrete computation of (5) in the case that $G_{N}=S U(N)$, Garoufalidis, Le, and Mariño gave an explicit presentation of the $S U(N)$ free energy of the lens space $L(d, b)$ :

Theorem 3 ([4, Theorem 1.4] ). The $S U(N)$ free energy of the lens space $L(d, b)$ is presented by

$$
F_{L(d, b), g}^{S U(N)}(\tau)= \begin{cases}(g-1) \frac{B_{g}}{g !}\left(d^{2-g} \operatorname{Li}_{3-g}\left(e^{\tau / d}\right)-\mathrm{Li}_{3-g}\left(e^{\tau}\right)\right)+a_{g}(\tau), & \text { if } g \text { is even, } \\ 0 & \text { if } g \text { is odd }\end{cases}
$$

where

$$
a_{g}(\tau)= \begin{cases}-\frac{\tau^{3}}{12}\left(d^{-1}-1\right)-\frac{\pi^{2} \tau}{6}(d-1)+\frac{\tau^{2}}{2} \log d+\left(d^{2}-1\right) \zeta(3)+\lambda_{L(d, b)} \frac{\tau^{3}}{2} & \text { if } g=0 \\ -\frac{\tau}{24}\left(d^{-1}-1\right)+\frac{1}{12} \log d-\lambda_{L(d, b)} \frac{\tau}{2} & \text { if } g=2, \\ 0 & \text { if } g \geq 4 .\end{cases}
$$


Here the $k$ th Bernoulli number $B_{k}$ is defined by the generating series

$$
\frac{x}{e^{x}-1}=\sum_{k=0}^{\infty} B_{k} \frac{x^{k}}{k !},
$$

and the polylogarithm function $\mathrm{Li}_{p}$ is defined by

$$
\operatorname{Li}_{p}(x):=\sum_{n=1}^{\infty} \frac{x^{n}}{n^{p}}
$$

for any integer $p$ and $\zeta(3):=\sum_{n=1}^{\infty} \frac{1}{n^{3}}$.

One sees that the power series $F_{L(d, b), g}^{S U(N)}(\tau)$ with even $g$ are analytic in a common neighborhood at zero, independently of $g$. Moreover, it is proved in [4] that for any closed 3-manifold $M$, the power series $F_{M, g}^{S U(N)}(\tau)$ with even $g$ is analytic in a neighborhood at zero, where the neighborhood can be chosen independently of $g$. They conjectured such analyticity of the $S O(N)$ and $S p(N)$ free energy of a closed oriented 3-manifold $M$, which was discussed in [5].

In the next section, when $G_{N}=S O(N)$ or $S p(N)$, by a concrete computation of the second term in the formula (5), we show their conjecture for lens spaces.

\section{Results}

In this section, we give an explicit presentation of the $S O(N)$ and $S p(N)$ free energy for lens spaces and show that the genus $g$ terms of $S O(N)$ and $S p(N)$ free energy for a rational homology 3 -sphere agree up to sign.

We have

Theorem 4. The $S O(N)$ and $S p(N)$ free energy of the lens space $L(d, b)$ is presented by

$$
F_{L(d, b), g}^{G_{N}}(\tau)=\left\{\begin{aligned}
\frac{1}{2}\left\{(g-1) \frac{B_{g}}{g !}\left(d^{2-g} \operatorname{Li}_{3-g}\left(e^{\tau / d}\right)-\mathrm{Li}_{3-g}\left(e^{\tau}\right)\right)+a_{g}(\tau)\right\} & \text { if } g \text { is even, } \\
\varepsilon_{G_{N}}\left[\frac { ( 2 ^ { g - 2 } - 1 ) B _ { g - 1 } } { ( g - 1 ) ! } \left\{d^{2-g}\left(2^{2-g} \operatorname{Li}_{3-g}\left(e^{\tau / 2 d}\right)-\frac{1}{2} \operatorname{Li}_{3-g}\left(e^{\tau / d}\right)\right)\right.\right. & \\
\left.\left.-2^{2-g} \operatorname{Li}_{3-g}\left(e^{\tau / 2}\right)+\frac{1}{2} \operatorname{Li}_{3-g}\left(e^{\tau}\right)\right\}+a_{g}^{\prime}(\tau)\right] & \text { if } g \text { is odd },
\end{aligned}\right.
$$

where $\varepsilon_{G_{N}}$ is 1 for $G_{N}=S O(N)$ and -1 for $G_{N}=S p(N)$,

$$
\begin{aligned}
& a_{g}(\tau)= \begin{cases}-\frac{\tau^{3}}{12}\left(d^{-1}-1\right)-\frac{\pi^{2} \tau}{6}(d-1)+\frac{\tau^{2}}{2} \log d+\left(d^{2}-1\right) \zeta(3)+\lambda_{L(d, b)} \frac{\tau^{3}}{2} & \text { if } g=0, \\
-\frac{\tau}{24}\left(d^{-1}-1\right)+\frac{1}{12} \log d-\lambda_{L(d, b)} \frac{\tau}{2} & \text { if } g=2, \\
0 & \text { if } g \geq 4,\end{cases} \\
& a_{g}^{\prime}(\tau)= \begin{cases}\frac{\tau}{2} \log d-\frac{\pi^{2}}{4}(d-1) & \text { if } g=1, \\
0 & \text { if } g \geq 3 .\end{cases}
\end{aligned}
$$

In particular, $F_{L(d, b), g}^{S O(N)}(\tau)$ and $F_{L(d, b), g}^{S p(N)}(\tau)$ are analytic in a neighborhood at zero, where we can choose the neighborhood independently of $g$. 
Proof. In the case that $G_{N}=S O(N)$ with even $N=2 n$, we show the required formula by calculating the right-hand side of (5) as follows.

The first term of the right-hand side of (5) is given by

$$
\frac{\lambda_{L(d, b)}}{4} C_{\mathfrak{s o}_{N}} \cdot \operatorname{dim} \mathfrak{s o}_{N} \cdot h=\frac{\lambda_{L(d, b)}}{4} N(N-1)(N-2) h=\frac{\lambda_{L(d, b)}}{4}\left(\frac{\tau^{3}}{h^{2}}-\tau\right),
$$

where $\tau=(N-1) h$.

We calculate the second term of the right-hand side of (5). For $j \in \mathbb{N}$, let $m(j)$ be the number of positive roots $\alpha$ such that $(\alpha, \rho)=j$. By definition,

$$
\sum_{\alpha \in \Psi_{+}} f((\alpha, \rho) h)=\sum_{j \in \mathbb{N}} m(j) f(j h) .
$$

Further, by Lemma 5 below,

$$
\begin{aligned}
\sum_{\alpha \in \Psi_{+}} f((\alpha, \rho) h)= & \sum_{\substack{j: \text { odd } \\
1 \leq j \leq n-1}} \frac{2 n-j+1}{2} f(j h)+\sum_{\substack{j: \text { even } \\
1 \leq j \leq n-1}} \frac{2 n-j}{2} f(j h) \\
& +\sum_{\substack{j: \text { odd } \\
n \leq j \leq 2 n-3}} \frac{2 n-j-1}{2} f(j h)+\sum_{\substack{j: \text { ven } \\
n \leq j \leq 2 n-3}} \frac{2 n-j-2}{2} f(j h) .
\end{aligned}
$$

From the definition of sinh, we have the following presentation of $f(x)$,

$$
f(x)=\sum_{k=1}^{\infty} \frac{B_{2 k}}{2 k(2 k) !} x^{2 k},
$$

where $B_{k}$ is the $k$ th Bernoulli number. So, it follows that

$$
\begin{aligned}
& \sum_{\alpha \in \Psi_{+}} f((\alpha, \rho) h) \\
& =\sum_{k=1}^{\infty} \frac{B_{2 k}}{2 k(2 k) !} h^{2 k}\left\{\sum_{1 \leq j \leq 2 n-2} \frac{2 n-j-1}{2} j^{2 k}+\sum_{1 \leq j \leq n-1} j^{2 k}-\frac{1}{2} \sum_{\substack{j \leq \text { even } \\
1 \leq j \leq 2 n-2}} j^{2 k}\right\} \\
& =\sum_{k=1}^{\infty} \frac{B_{2 k}}{2 k(2 k) !} h^{2 k} \sum_{1 \leq j \leq 2 n-2} \frac{2 n-j-1}{2} j^{2 k}+\sum_{k=1}^{\infty} \frac{B_{2 k}}{2 k(2 k) !} h^{2 k}\left(1-2^{2 k-1}\right) \sum_{1 \leq j \leq n-1} j^{2 k} .
\end{aligned}
$$

By using $2 n-1=N-1=\tau / h$, from the formulas (6.7), (6.8), and (6.10) in [4], the first term of (7) is presented by

$$
\begin{aligned}
\sum_{k=1}^{\infty} \frac{B_{2 k}}{2 k(2 k) !} h^{2 k} \sum_{1 \leq j \leq 2 n-2} \frac{2 n-j-1}{2} j^{2 k} & =\frac{1}{2} \sum_{s=0}^{\infty} \frac{(1-2 s) B_{2 s} h^{2 s-2}}{(2 s) !} \sum_{l=0}^{\infty} \frac{B_{2 l+2 s}}{(2 l+2) !(2 l+2 s)} \tau^{2 l+2} \\
& =\frac{1}{2} \sum_{s=0}^{\infty} \frac{(1-2 s) B_{2 s} h^{2 s-2}}{(2 s) !} F_{s}^{\text {even }}(\tau),
\end{aligned}
$$

where we put

$$
F_{s}^{\text {even }}(\tau):=\sum_{l=0}^{\infty} \frac{B_{2 l+2 s}}{(2 l+2) !(2 l+2 s)} \tau^{2 l+2} .
$$


Using the formula

$$
\sum_{j=1}^{n} j^{2 k}=\frac{\left(n+\frac{1}{2}\right)^{2 k+1}}{2 k+1}+\sum_{s=1}^{k} \frac{2^{1-2 s}-1}{2 k+1}\left(\begin{array}{c}
2 k+1 \\
2 g
\end{array}\right) B_{2 s}\left(n+\frac{1}{2}\right)^{2 k+1-2 s},
$$

the second term of (7) is presented by

$$
\begin{aligned}
& \sum_{k=1}^{\infty} \frac{B_{2 k}}{2 k(2 k) !} h^{2 k}\left(1-2^{2 k-1}\right) \sum_{1 \leq j \leq n-1} j^{2 k} \\
& =\sum_{k=1}^{\infty} \frac{B_{2 k}}{2 k(2 k) !} h^{2 k}\left(1-2^{2 k-1}\right)\left\{\frac{\left(n-\frac{1}{2}\right)^{2 k+1}}{2 k+1}+\sum_{s=1}^{k} \frac{2^{1-2 s}-1}{2 k+1}\left(\begin{array}{c}
2 k+1 \\
2 s
\end{array}\right) B_{2 s}\left(n-\frac{1}{2}\right)^{2 k+1-2 s}\right\} \\
& =\sum_{k=1}^{\infty} \frac{B_{2 k}}{2 k(2 k) !} h^{2 k}\left(1-2^{2 k-1}\right) \\
& \times\left\{\frac{(2 n-1)^{2 k+1}}{2 k+1}\left(\frac{1}{2}\right)^{2 k+1}+\sum_{s=1}^{k} \frac{2^{1-2 s}-1}{2 k+1}\left(\begin{array}{c}
2 k+1 \\
2 s
\end{array}\right) B_{2 s}(2 n-1)^{2 k+1-2 s}\left(\frac{1}{2}\right)^{2 k+1-2 s}\right\} \\
& =h^{-1} \sum_{k=1}^{\infty} \frac{B_{2 k}}{2 k(2 k) !}\left(1-2^{2 k-1}\right) \frac{\tau^{2 k+1}}{2 k+1}\left(\frac{1}{2}\right)^{2 k+1} \\
& +\sum_{k=1}^{\infty} \frac{B_{2 k}}{2 k(2 k) !} h^{2 g-1}\left(1-2^{2 k-1}\right) \sum_{s=1}^{k} \frac{2^{1-2 s}-1}{2 k+1}\left(\begin{array}{c}
2 k+1 \\
2 s
\end{array}\right) B_{2 s} \tau^{2 k+1-2 s}\left(\frac{1}{2}\right)^{2 k+1-2 s} \\
& =h^{-1}\left(\sum_{k=1}^{\infty} \frac{B_{2 k}}{2 k(2 k+1) !}\left(\frac{\tau}{2}\right)^{2 k+1}-\sum_{k=1}^{\infty} \frac{B_{2 k}}{2 k(2 k+1) !}\left(\frac{1}{2}\right)^{2} \tau^{2 k+1}\right) \\
& +\sum_{s=1}^{\infty} \frac{\left(2^{1-2 s}-1\right) B_{2 s}}{(2 s) !} h^{2 s-1} \\
& \times\left(\sum_{k=s}^{\infty} \frac{B_{2 k}}{2 k(2 k+1-2 s) !}\left(\frac{\tau}{2}\right)^{2 k+1-2 s}-\left(\frac{1}{2}\right)^{2-2 s} \sum_{k=s}^{\infty} \frac{B_{2 k}}{2 k(2 k+1-2 s) !} \tau^{2 k+1-2 s}\right) \\
& =h^{-1}\left(\sum_{k=1}^{\infty} \frac{B_{2 k}}{2 k(2 k+1) !}\left(\frac{\tau}{2}\right)^{2 k+1}-\left(\frac{1}{2}\right)^{2} \sum_{k=1}^{\infty} \frac{B_{2 k}}{2 k(2 k+1) !} \tau^{2 k+1}\right) \\
& +\sum_{s=1}^{\infty} \frac{\left(2^{1-2 s}-1\right) B_{2 s}}{(2 s) !} h^{2 s-1} \\
& \times\left(\sum_{l=0}^{\infty} \frac{B_{2 l+2 s}}{(2 l+1) !(2 l+2 s)}\left(\frac{\tau}{2}\right)^{2 l+1}-\left(\frac{1}{2}\right)^{2-2 s} \sum_{l=0}^{\infty} \frac{B_{2 l+2 s}}{(2 l+1) !(2 l+2 s)} \tau^{2 l+1}\right) \\
& =\sum_{s=0}^{\infty} \frac{\left(1-2^{2 s-1}\right) B_{2 s}}{(2 s) !} h^{2 s-1}\left(2^{1-2 s} F_{s}^{o d d}(\tau / 2)-\frac{1}{2} F_{s}^{o d d}(\tau)\right),
\end{aligned}
$$

where we put

$$
F_{s}^{o d d}(\tau):=\sum_{l=0}^{\infty} \frac{B_{2 l+2 s}}{(2 l+2 s)(2 l+1) !} \tau^{2 l+1}
$$


Thus, it turns out that

$$
\begin{aligned}
& \sum_{\alpha \in \Psi_{+}} f((\alpha, \rho) h) \\
& =\frac{1}{2} \sum_{s=0}^{\infty} \frac{(1-2 s) B_{2 s} h^{2 s-2}}{(2 s) !} F_{s}^{e v e n}(\tau)+\sum_{s=0}^{\infty} \frac{\left(1-2^{2 s-1}\right) B_{2 s}}{(2 s) !} h^{2 s-1}\left(2^{1-2 s} F_{s}^{o d d}(\tau / 2)-\frac{1}{2} F_{s}^{o d d}(\tau)\right) .
\end{aligned}
$$

Hence, from the formula (5), with the replacement of $(\tau, h)$ to $(\tau / d, h / d)$, we obtain

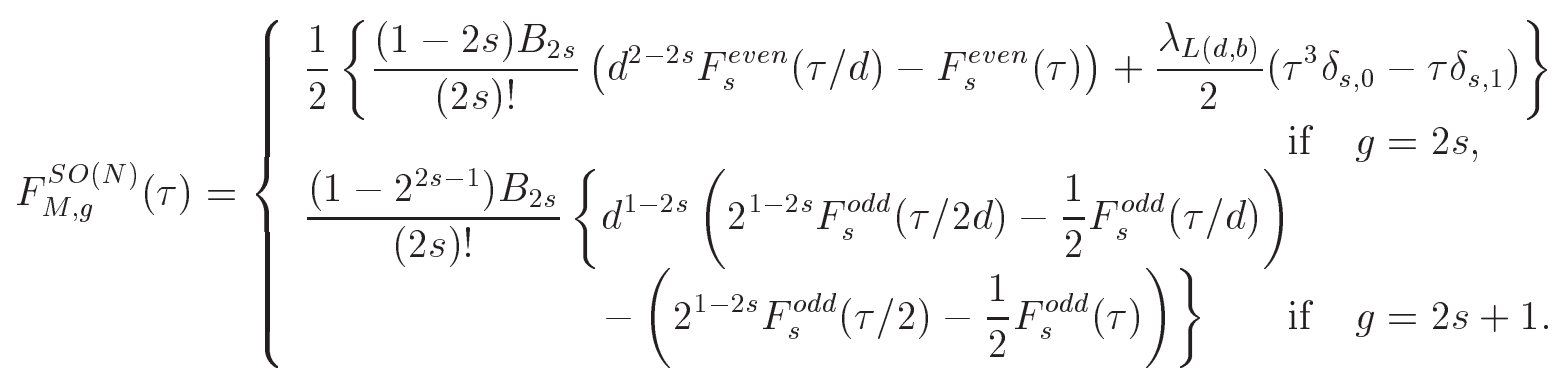

From Lemma 7 below, we obtain the required formula for $G_{N}=S O(N)$ with even $N$.

In the case that $G_{N}=S O(N)$ with odd $N=2 n+1$, from Lemma 6 below, it follows that

$$
\sum_{\alpha \in \Psi_{+}} f((\alpha, \rho) h)=\sum_{k=1}^{\infty} \frac{B_{2 k}}{2 k(2 k) !} h^{2 k}\left\{\sum_{1 \leq j \leq 2 n-1} \frac{2 n-j}{2} j^{2 k}+\frac{1-2^{2 k-1}}{2^{2 k}} \sum_{\substack{j: \text { odd } \\ 1 \leq j \leq 2 n-1}} j^{2 k}\right\} .
$$

By a similar calculation, we obtain the required formula for $G_{N}=S O(N)$ with odd $N=2 n+1$.

We obtain the required formula for $G_{N}=S p(N)$, since

$$
F_{L(d, b), g}^{S p(N)}(\tau)=(-1)^{g} F_{L(d, b), g}^{S O(N)}(\tau),
$$

by Proposition 8 below.

In particular, we see that for any $g, F_{L(d, b), g}^{S O(N)}(\tau)$ and $F_{L(d, b), g}^{S p(N)}(\tau)$ are analytic in the unit disk, which is not trivial, since the function $\operatorname{Li}_{3-g}\left(e^{\tau}\right)$ for $g \geq 4$ has poles at $2 \pi \sqrt{-1} \mathbb{Z}$. Hence, $F_{L(d, b), g}^{S O(N)}(\tau)$ and $F_{L(d, b), g}^{S p(N)}(\tau)$ are analytic in a neighborhood of zero, where we can choose the neighborhood independently of $g$.

Lemma 5. For $j \in \mathbb{N}$, let $m(j)$ be the number of positive roots $\alpha$ of $\mathfrak{s o}_{2 n}$ such that $(\alpha, \rho)=j$. We have that

$$
m(j)= \begin{cases}\frac{2 n-j+1}{2} & \text { if } j: \text { odd, } 1 \leq j \leq n-1, \\ \frac{2 n-j}{2} & \text { if } j: \text { even, } 1 \leq j \leq n-1, \\ \frac{2 n-j-1}{2} & \text { if } j: \text { odd, } n \leq j \leq 2 n-3, \\ \frac{2 n-j-2}{2} & \text { if } j: \text { even, } n \leq j \leq 2 n-3, \\ 0 & \text { otherwise. }\end{cases}
$$

Proof. The set of positive roots of $\mathfrak{s o}_{2 n}$ is

$$
\Psi_{+}=\left\{\varepsilon_{k} \pm \varepsilon_{l} \mid 1 \leq k<l \leq n\right\},
$$

$\left(\varepsilon_{k}, \varepsilon_{l}\right)=\delta_{k l}$, and $\rho=\sum_{k=1}^{n-1}(n-k) \varepsilon_{k}$. Since $\left(\varepsilon_{k}-\varepsilon_{l}, \rho\right)=l-k$ for $1 \leq k<l \leq n$, it holds that for $j \in \mathbb{N}$, the number of $\varepsilon_{k}-\varepsilon_{l}$ with $\left(\varepsilon_{k}-\varepsilon_{l}, \rho\right)=j$ is $n-j$ if $1 \leq j \leq n-1$ and 0 otherwise. 
Since $\left(\varepsilon_{k}+\varepsilon_{l}, \rho\right)=2 n-k-l$ for $1 \leq k<l \leq n$, it holds that for $j \in \mathbb{N}$, the number of $\varepsilon_{k}+\varepsilon_{l}$ with $\left(\varepsilon_{k}+\varepsilon_{l}, \rho\right)=j$ is

$$
\begin{array}{ll}
\frac{j+1}{2} & \text { if } j: \text { odd, } 1 \leq j \leq n-1, \\
\frac{j}{2} & \text { if } j: \text { even, } 1 \leq j \leq n-1, \\
\frac{2 n-j-1}{2} & \text { if } j: \text { odd, } n \leq j \leq 2 n-3, \\
\frac{2 n-j-2}{2} & \text { if } j: \text { even, } n \leq j \leq 2 n-3, \\
0 & \text { otherwise. }
\end{array}
$$

Then, we obtain the required formula.

Lemma 6. For $j \in \mathbb{N}$, let $m(j)$ be the number of positive roots $\alpha$ of $\mathfrak{s o}_{2 n+1}$ such that $(\alpha, \rho)=j$. We have that

$$
m(j)= \begin{cases}1 & \text { if } j=\frac{2 l-1}{2}, 1 \leq l \leq n, \\ \frac{2 n-j-1}{2} & \text { if } j: \text { odd, } n+1 \leq j \leq 2 n-1 \\ \frac{2 n-j}{2} & \text { if } j: \text { even, } n+1 \leq j \leq 2 n-2 \\ 0 & \text { otherwise }\end{cases}
$$

Lemma 7. We have

$$
\begin{aligned}
& F_{s}^{\text {even }}(\tau)=-\operatorname{Li}_{3-2 s}\left(e^{\tau}\right)+ \begin{cases}-\frac{\tau^{2}}{2} \log (-\tau)-\frac{\tau^{3}}{12}+\frac{3 \tau^{2}}{4}-\frac{\pi^{2} \tau}{6}+\zeta(3) & \text { if } s=0, \\
-\log (-\tau)-\frac{\tau}{2} & \text { if } s=1, \\
(2 s-3) ! \tau^{2-2 s}-\frac{B_{2 s-2}}{2 s-2} & \text { if } s \geq 2,\end{cases} \\
& F_{s}^{\text {odd }}(\tau)=-\operatorname{Li}_{2-2 s}\left(e^{\tau}\right)+ \begin{cases}-\tau \log (-\tau)-\frac{1}{4} \tau^{2}-\frac{\pi^{2}}{6}+\tau & \text { if } s=0, \\
-\frac{1}{\tau}-\frac{1}{2} & \text { if } s=1, \\
-(2 s-2) ! \tau^{1-2 s} & \text { if } s \geq 2 .\end{cases}
\end{aligned}
$$

Proof. The first formula follows from [4], by noting that $F_{s}^{\text {even }}(\tau)$ equals (6.8) in [4]. As $F_{s}^{o d d}(\tau)=\partial_{\tau} F_{s}^{\text {even }}(\tau)$ and $\partial_{\tau} \operatorname{Li}_{p}\left(e^{\tau}\right)=\operatorname{Li}_{p-1}\left(e^{\tau}\right)$ for any integer $p$, the second formula follows from the first formula.

We show a relation between the genus $g$ terms of $S O(N)$ and $S p(N)$ free energy for a rational homology 3-sphere, which we used in the proof of Theorem 4.

Proposition 8. For any rational homology 3-sphere $M$ and any $g$,

$$
F_{M, g}^{S p(N)}(\tau)=(-1)^{g} F_{M, g}^{S O(N)}(\tau) .
$$

Proof. Noting that $\tau=N-1$ for $\mathfrak{g}=\mathfrak{s o}$ and that $\tau=N+1$ for $\mathfrak{g}=\mathfrak{s p}$, it follows from (2) that

$$
\begin{aligned}
& W_{\mathfrak{s p}_{\star}}(D)=\sum_{0 \leq g \leq d+1} c_{\mathfrak{s p}, g}(D)(N+1)^{d+2-g} h^{g-2}, \\
& W_{\mathfrak{s o}_{\star}}(D)=\sum_{0 \leq g \leq d+1} c_{\mathfrak{s o}, g}(D)(N-1)^{d+2-g} h^{g-2}
\end{aligned}
$$

for a connected trivalent graph $D$ of degree $d$. Hence,

$$
\left.(-1)^{d} W_{\mathfrak{s o}_{\star}}(D)\right|_{N \rightarrow-N}=(-1)^{d} \sum_{\substack{0 \leq g \leq d+1 \\ 8}} c_{\mathfrak{s o}, g}(D)(-N-1)^{d+2-g} h^{g-2}
$$




$$
=\sum_{0 \leq g \leq d+1}(-1)^{g} c_{\mathfrak{s o}, g}(D)(N+1)^{d+2-g} h^{g-2} .
$$

Comparing $W_{\mathfrak{s p}_{\star}}(D)$ and $\left.(-1)^{d} W_{\mathfrak{s o}_{\star}}(D)\right|_{N \rightarrow-N}$ by Proposition 9 below, we have

$$
c_{\mathfrak{s p}, g}(D)=(-1)^{g} c_{\mathfrak{s o}, g}(D)
$$

for any $g$. Since $\log Z_{M}$ is a linear sum of such $D$, it follows from (3) that

$$
c_{\mathfrak{s p}, d, g}(M)=(-1)^{g} c_{\mathfrak{s o}, d, g}(M)
$$

for any rational homology 3 -sphere $M$, any $d$, and any $g$. Further, since

$$
F_{M, g}^{G_{N}}(\tau)=\sum_{d>0, d \geq g-1} c_{\mathfrak{g}, d, g}(M) \tau^{d+2-g}
$$

by definition, we obtain the required formula.

Proposition 9. For a connected trivalent graph $D$ of degree $d, W_{\mathfrak{s p}_{N}}(D)$ is obtained from $(-1)^{d} W_{\mathfrak{s o}_{N}}(D)$ by replacing $N$ with $-N$, i.e., $W_{\mathfrak{s p}_{N}}(D)=\left.(-1)^{d} W_{\mathfrak{s o}_{N}}(D)\right|_{N \rightarrow-N}$.

This proposition was proved up to sign in [3, Chapter 13], while we give a complete proof in another way in Section 5. As a corollary of Theorems 3 and 4, we obtain

Corollary 10. For the lens space $L(d, b)$ and any even $g$,

$$
\frac{1}{2} F_{L(d, b), g}^{S U(N)}(\tau)=F_{L(d, b), g}^{S O(N)}(\tau)=F_{L(d, b), g}^{S p(N)}(\tau) .
$$

Proof. The first equality follows from Theorems 3 and 4 and the second equality follows from Proposition 8.

\section{Observation}

In this section, we review the descriptions of $W_{\mathfrak{s l}_{N}}$ and $W_{\mathfrak{s o}_{N}}$ given by Bar-Natan in $[1,2]$ and observe new weight systems related to the free energy.

We consider the weight system $W_{\mathfrak{s l}_{N}}$. We double any edge and replace any trivalent vertex of $D$ in the following:

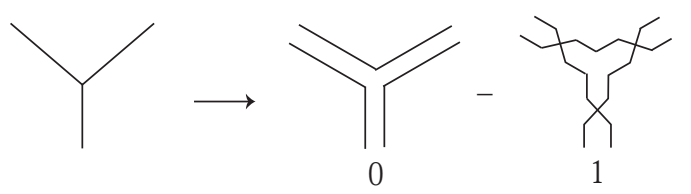

FiguRE 1

This diagrammatic interpretation comes from the fact that $\mathfrak{g l}_{N}=V \otimes V^{*}$ for the defining representation $V$ of $\mathfrak{g l}_{N}$ and the $\mathfrak{g l}_{N}$ weight system at a trivalent vertex is defined by the Lie bracket. We note that the $\mathfrak{g l}_{N}$ and $\mathfrak{s l}_{N}$ weight systems agree on a trivalent graph, since an abelian ideal of $\mathfrak{g l}_{N}$ does not contribute on any trivalent vertex applied with the $\mathfrak{g l}_{N}$ weight system. Let $D$ be a connected trivalent graph and $v(D)$ the set of trivalent vertices. Given a map $m_{v}: v(D) \rightarrow\{0,1\}$, called a vertex marking of $D$, choosing one of the two possibilities for the replacement of a trivalent vertex depending on $m_{v}$, connecting up, we obtain an orientable 
surface $S_{D, m_{v}}$ of the genus $g\left(S_{D, m_{v}}\right)$ with $b_{D, m_{v}}$ boundary components. It is showed that for a connected trivalent graph $D$ of degree $d$,

$$
W_{\mathfrak{s l}_{N}}(D)=\sum_{m_{v}}(-1)^{s_{m_{v}}} N^{b_{D, m_{v}}} h^{d},
$$

where $s_{m_{v}}=\sum_{x \in v(D)} m_{v}(x)$ and the sum is over all possible vertex marking $m_{v}$ of $D$. On the other hand, It holds that $2-2 g\left(S_{D, m_{v}}\right)=\chi(D)+b_{D, m_{v}}$, where $\chi(D)$ denotes the Euler characteristic of $D$. As the degree of $D$ is a half of the number of trivalent vertices and $\chi(D)=-d$, we get

$$
W_{\mathfrak{s l}_{N}}(D)=\sum_{m_{v}}(-1)^{s_{m_{v}}} N^{d+2-2 g\left(S_{D, m_{v}}\right)} h^{d}
$$

For example, if $D=x_{1} \bigcirc x_{2}$ and $m_{v}\left(x_{1}\right)=0, m_{v}\left(x_{2}\right)=1$, then $s_{m_{v}}=1$ and $S_{D, m_{v}}=\bigcirc$ is a torus with one boundary component, i.e., $g\left(S_{D, m_{v}}\right)=1, b_{D, m_{v}}=1$. This contributes $-N h$ to $W_{\mathfrak{s l}_{N}}(D)$. We get that $W_{\mathfrak{g l}_{N}}(D)=2 N^{3} h-2 N h=2 N\left(N^{2}-1\right) h$.

Moreover, we have the following description of the weight system $W_{\mathfrak{s o}_{N}}$. We replace any trivalent vertex and any edge in the following:

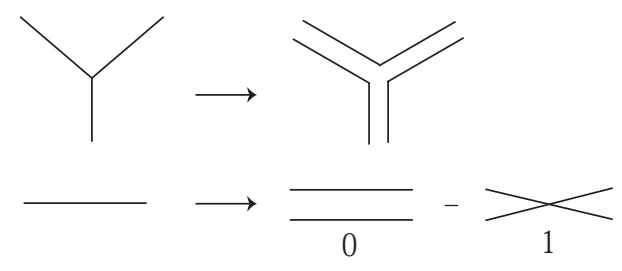

Figure 2

We denote by $e(D)$ the set of edges of a connected trivalent graph $D$. Given a map $m_{e}: e(D) \rightarrow\{0,1\}$, called an edge marking of $D$, choosing one of the two possibilities for the replacement of an edge depending on $m_{e}$, connecting up, we obtain an orientable or a nonorientable surface $S_{D, m_{e}}$ of the genus $g\left(S_{D, m_{e}}\right)$ with $b_{D, m_{e}}$ boundary components. Then, we have

$$
W_{\mathfrak{s o}_{N}}(D)=\sum_{m_{e}}(-1)^{s_{m_{e}}} N^{b_{D, m_{e}}} h^{d}=\sum_{m_{v}}(-1)^{s_{m_{v}}} N^{d+2-g^{\prime}{ }_{D, m_{v}}} h^{d}
$$

where $s_{m_{e}}=\sum_{y \in e(D)} m_{e}(y)$, the sum is over all possible edge marking $m_{e}$ of $D$, and $g^{\prime}{ }_{D, m_{e}}=$ $2 g\left(S_{D, m_{e}}\right)$ if the surface $S_{D, m_{e}}$ is orientable and $g_{D, m_{e}}^{\prime}=g\left(S_{D, m_{e}}\right)$ if the surface $S_{D, m_{e}}$ is nonorientable. For example, from $\frac{1}{0}$, we obtain $S_{D, m_{e}}=\bigcirc$ is a projective plane with two boundary components. This contributes $-N^{2} h$ to $W_{\mathfrak{s o}_{N}}(\ominus)$. We get that $W_{\mathfrak{s o}_{N}}(\theta)=$ $N^{3} h-3 N^{2} h+3 N h-N h=N(N-1)(N-2) h$. We remark that the inner product for $\mathfrak{s o}_{N}$ here is the one in [2] multiplied by $\frac{1}{2}$.

Using the above descriptions of $W_{\mathfrak{s l}_{N}}$ and $W_{\mathfrak{s o}_{N}}$, we show Lemma 1.

Proof of Lemma 1 By noting that $b_{D, m_{v}}>0$ in (8) and that $b_{D, m_{e}}>0$ in (10), Lemma 1 follows from the above descriptions (9) and (10) and Proposition 9. 
Let us observe new weight systems related to the $G_{N}$ free energy. We recall the presentation (2) of $W_{\mathfrak{g}_{\star}}(D)$ for $\mathfrak{g}=\mathfrak{s l}, \mathfrak{s o}, \mathfrak{s p}$ and a connected trivalent graph $D$ of degree $d$,

$$
W_{\mathfrak{g}_{\star}}(D)=\sum_{0 \leq g \leq d+1} c_{\mathfrak{g}, g}(D) \tau^{d+2-g} h^{g-2},
$$

for some $c_{\mathfrak{g}, g}(D) \in \mathbb{Z}$. For $\mathfrak{g}=\mathfrak{s l}, \mathfrak{s o}, \mathfrak{s p}$ and any $g$, we get the weight system $w_{\mathfrak{g}_{\star}, g}: \mathcal{A}(\emptyset)_{\text {conn }} \rightarrow$ $\mathbb{Q}[[\tau]]$ defined by

$$
w_{\mathfrak{g}_{*}, g}(D):= \begin{cases}c_{\mathfrak{g}, g}(D) \tau^{d+2-g} & \text { if } d \geq g-1 \\ 0 & \text { otherwise }\end{cases}
$$

for a connected trivalent graph $D$ of degree $d$.

We study relations among the weight systems $w_{\mathfrak{s l}_{\star}, g}, w_{\mathfrak{s o}_{\star}, g}$ and $w_{\mathfrak{s l}_{\star}, g}$. Since only orientable surface appears in the above description of the weight system $W_{\mathfrak{s l}_{N}}, w_{\mathfrak{s l}_{\star}, g} \equiv 0$ for any odd $g$, and Proposition 9 implies

Proposition 11. For any connected trivalent graph $D$ and any $g$,

$$
w_{\mathfrak{s o}_{\star}, g}(D)=(-1)^{g} w_{\mathfrak{s p}_{\star}, g}(D) .
$$

We consider the weight systems $w_{\mathfrak{s l}_{\star}, g}$ for even $g$ and $w_{\mathfrak{s o}_{\star}, g}$ for any $g$. In the case that $g=0$, we have

Proposition 12. For any connected trivalent graph D,

$$
w_{\mathfrak{s o}_{\star}, 0}(D)=\frac{1}{2} w_{\mathfrak{s}_{\star}, 0}(D) .
$$

Proof. One sees that two different vertex markings $m_{v}$ and $m_{v}^{\prime}$ of $D$ induce the same edge marking of $D$ if and only if $m_{v}^{\prime}(x)-m_{v}(x)=1(\bmod 2)$ for any vertex $x$ of $D$. Conversely, if an edge marking $m_{e}$ of $D$ gives an orientable surface, then there exists a vertex marking of $D$ which induces the edge marking $m_{e}$. Noting that only edge marking of $D$ such that gives orientable surface contributes to $w_{\mathfrak{s o}_{N}, 0}(D)$, we obtain the required formula.

Moreover, we obtain

Proposition 13. The family $\left\{w_{\mathfrak{s l}_{\star}, g} \mid g\right.$ is even, $\left.g>0\right\} \cup\left\{w_{\mathfrak{s o}_{\star}, g} \mid g \geq 0\right\}$ of the weight systems are linearly independent in the space spanned over $\mathbb{Q}$ by these weight systems.

To show Proposition 13, we need some lemmas. We define $t D$ ( resp. $u D$ ) for a connected trivalent graph $D$ in $\mathcal{A}(\emptyset)_{\text {conn }}$ to be a connected trivalent graph obtained by replacing a trivalent vertex in $D$ with $\lambda$ ( resp. 1 ), which was introduced by Vogel in [9]. By the AS and IHX relations, these replacements are independent of a choice of a trivalent vertex. We have the following lemma (for example, see [9]).

Lemma 14. For any simple Lie algebra $\mathfrak{g}$ and any connected trivalent graph $D$ in $\mathcal{A}(\emptyset)_{\mathrm{conn}}$,

$$
W_{\mathfrak{g}}\left(-\bigcirc_{-}\right)=C_{\mathfrak{g}} W_{\mathfrak{g}}(-), W_{\mathfrak{g}}(t D)=\frac{1}{2} C_{\mathfrak{g}} W_{\mathfrak{g}}(D),
$$

where $C_{\mathfrak{g}}$ is the quadratic Casimir of $\mathfrak{g}$.

From Lemma 14, we also have 
Lemma 15. For any simple Lie algebra $\mathfrak{g}$ and any connected trivalent graph $D$ in $\mathcal{A}(\emptyset)_{\mathrm{conn}}$,

$$
W_{\mathfrak{g}}(u D)=\frac{W_{\mathfrak{g}}(\bigoplus)}{W_{\mathfrak{g}}(\bigoplus)} W_{\mathfrak{g}}(D)=\frac{\sum d_{i} \alpha_{i}^{4}}{C_{\mathfrak{g}} \operatorname{dim} \mathfrak{g}} W_{\mathfrak{g}}(D) .
$$

Here $\left\{\alpha_{i}\right\}$ are the eigenvalues of the $\mathfrak{g}$-homomorphism from $\mathfrak{g} \otimes \mathfrak{g}$ to itself defined by $x \otimes y \mapsto$ $\sum_{\alpha}\left[x, \mathfrak{g}_{\alpha}\right] \otimes\left[\mathfrak{g}_{\alpha}^{\prime}, y\right]$ with the Casimir element $\sum_{\alpha} \mathfrak{g}_{\alpha} \mathfrak{g}_{\alpha}^{\prime}$, where $\left\{\mathfrak{g}_{\alpha}\right\}$ is a basis of $\mathfrak{g},\left\{\mathfrak{g}_{\alpha}^{\prime}\right\}$ is the dual basis on the Killing form, and $d_{i}$ is the dimension of the eigenspace of $\alpha_{i}$.

Proof. From Lemma 14, for $\downarrow=\varnothing$, there exists a scalar $\lambda_{\mathfrak{g}}$ such that

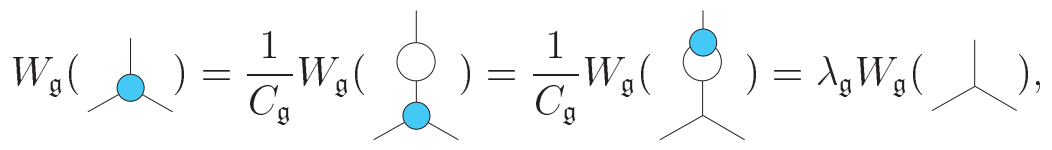

and so $W_{\mathfrak{g}}(u D)=\lambda_{\mathfrak{g}} W_{\mathfrak{g}}(D)$. Applying to this $D=\bigoplus$, we get that

$$
\lambda_{\mathfrak{g}}=W_{\mathfrak{g}}(\Theta) / W_{\mathfrak{g}}(\ominus) .
$$

The second equality can be obtained from [9, Proposition 6.2].

Using Lemmas 14 and 15, we get the following lemma.

Lemma 16. For the connected trivalent graph $T_{m, n}:=t^{m} u^{n} \bigcirc$ of degree $m+3 n+1$,

$$
\begin{aligned}
W_{\mathfrak{s l}_{\star}}\left(T_{m, n}\right) & =2 N^{m+n+1}\left(N^{2}+12\right)^{n}\left(N^{2}-1\right) h^{m+3 n+1} \\
& =2 \tau^{m+n+1}\left(\left(\frac{\tau}{h}\right)^{2}+12\right)^{n}\left(\left(\frac{\tau}{h}\right)^{2}-1\right) h^{2 n}, \\
W_{\mathfrak{s o}_{\star}}\left(T_{m, n}\right) & =(N-2)^{m+1}\left(N^{3}-9 N^{2}+54 N-104\right)^{n} N(N-1) h^{m+1} \\
& =\tau\left(\frac{\tau}{h}-1\right)^{m}\left(\left(\frac{\tau}{h}\right)^{3}-6\left(\frac{\tau}{h}\right)^{2}+39\left(\frac{\tau}{h}\right)-58\right)^{n}\left(\left(\frac{\tau}{h}\right)^{2}-1\right) h^{m},
\end{aligned}
$$

where $\tau=N h$ for $W_{\mathfrak{s l}_{\star}}$ and $\tau=(N-1) h$ for $W_{\mathfrak{s o}_{\star}}$.

Proof. We have that $C_{\mathfrak{s l}_{N}}=2 N$ and $C_{\mathfrak{s o}_{N}}=2(N-2)$ and calculate

$$
\begin{aligned}
& W_{\mathfrak{s l}_{N}}(\bigoplus)=2 N\left(N^{2}-1\right), \\
& W_{\mathfrak{s l}_{N}}(\bigoplus)=2 N^{2}\left(N^{2}-1\right)\left(N^{2}+12\right), \\
& W_{\mathfrak{s o}_{N}}(\bigoplus)=N(N-1)(N-2), \\
& W_{\mathfrak{s v}_{N}}(\bigoplus)=N(N-1)(N-2)\left(N^{3}-9 N^{2}+54 N-104\right) .
\end{aligned}
$$

From Lemmas 14 and 15, we obtain the required formulas.

Now let us show Proposition 13.

Proof of Proposition 13 From Lemma 16, we calculate that for $g \geq 3$,

$$
\begin{aligned}
& w_{\mathfrak{s l}_{\star}, 2}\left(T_{g-2,0}\right)=-2 \tau^{g-1}, w_{\mathfrak{s I}_{\star}, m}\left(T_{g-2,0}\right)=0 \text { if } m \geq 4, m \text { is even, } \\
& w_{\mathfrak{s}_{\star}, 0}\left(T_{g-2,0}\right)=\tau^{g+1}, w_{\mathfrak{s o}_{\star}, 1}\left(T_{g-2,0}\right)=-(g-2) \tau^{g}, \\
& w_{\mathfrak{s o}_{\star}, 2}\left(T_{g-2,0}\right)=\frac{(g-1)(g-4)}{2} \tau^{g-1}, w_{\mathfrak{s o}_{\star}, 3}\left(T_{g-2,0}\right)=-\frac{(g-1)(g-2)(g-6)}{6} \tau^{g-2},
\end{aligned}
$$




$$
w_{\mathfrak{s o}_{\star}, g}\left(T_{g-2,0}\right)=(-1)^{g-1} \tau, w_{\mathfrak{s o}_{\star}, m}\left(T_{g-2,0}\right)=0 \text { if } m>g,
$$

and that for any even $g$ with $g \geq 4$,

$$
w_{\mathfrak{s l}_{\star}, g}\left(T_{0, \frac{g-2}{2}}\right)=-2 \cdot 12^{\frac{g-2}{2}} \tau^{\frac{g}{2}}, w_{\mathfrak{s} \mathfrak{I}_{\star}, m}\left(T_{0, \frac{g-2}{2}}\right)=0 \text { if } m \geq g+2, m \text { is even. }
$$

Then, we get the proposition.

\section{Proof of Proposition 9}

Let us state some results about the $\mathfrak{s p}_{N}$ weight system. From [1], we get the following diagrammatic description of the $\mathfrak{s p}_{N}$ weight system with $N=2 n$, which comes from that $\mathfrak{s p}_{N}$ has a basis $E_{i j}-E_{n+j n+i}(1 \leq i, j \leq n), E_{i n+j}+E_{j n+i}(1 \leq i \leq j \leq n)$, and $E_{n+i j}+E_{n+j i}$ $(1 \leq i \leq j \leq n)$. and that the inner product is given by $\left(E_{i j}, E_{k l}\right)=\frac{1}{2} \operatorname{tr}\left(E_{i j} E_{k l}\right)(1 \leq i, j, k, l \leq$ $2 n)$. Let $D$ be a connected trivalent graph, $v(D)$ the set of vertices of $D$, and $Y_{0}(\circ, \bullet)$ the set of the diagrams and the diagrams obtained by the $\frac{2 \pi}{3}$-rotation or $\frac{4 \pi}{3}$-rotation of the above

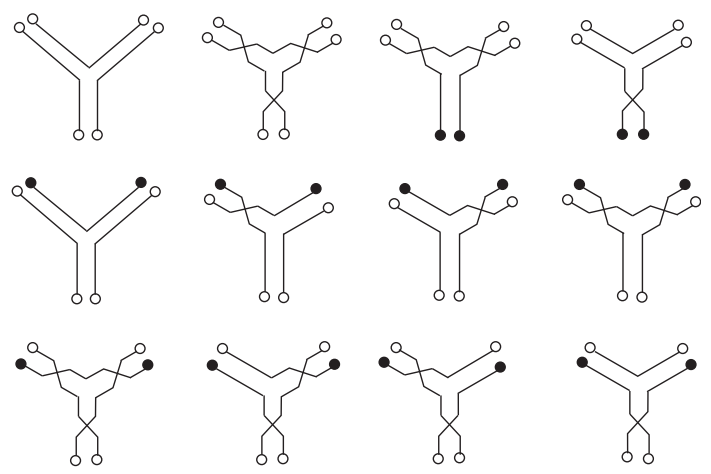

FiguRE 3

diagrams except the first and second diagrams. We double any edge in $D$ and replace each vertex with one diagram in $Y:=Y_{0}(\circ, \bullet) \cup Y_{0}(\bullet, \circ)$, in such a way that connecting up, the two ends of each edge in any double edge have the same symbol. Such a replacement defines a map $m: v(D) \rightarrow Y$, called an admissible vertex marking of $D$, and we obtain an orientable or a nonorientable surface $S_{D, m}$ with $b_{D, m}$ boundary components with even symbols $\circ$ and even symbols •. We comment that the symbol o ( resp. •) corresponds to index $i(\operatorname{resp} . n+i)$ with $1 \leq i \leq n$ in the above basis of $\mathfrak{s p}_{N}$. Then, we have

$$
W_{\mathfrak{s p}_{N}}(D)=2^{-3 d} \sum_{m}(-1)^{s_{m}} n^{b_{D, m}} h^{d},
$$

where $s_{m}$ is the number of $\chi_{0}^{\circ}$ and $\chi_{\text {: }}$ in $S_{D, m}$, and the sum is over all possible admissible vertex marking $m$ of $D$. We note that the symbols $\circ$ and $\bullet$ correspond to the symbols $P$ and $Q$ respectively in [1].

We have a simpler description of the weight system $W_{\mathfrak{s p}_{N}}$. We denote by $e(D)$ the set of edges of a connected trivalent graph $D$ and $Y^{\prime}$ the set of the diagrams We replace any trivalent

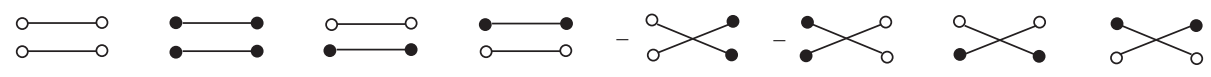

FiguRE 4 
vertex in the same way as the weight system $W_{\mathfrak{s o}_{N}}$ and replace each edge with one diagram in $Y^{\prime}$, in such a way that connecting up, the two ends of each arc in any doubled vertex have the same symbol. Such a replacement defines a map $m^{\prime}: e(D) \rightarrow Y^{\prime}$, called an admissible edge marking of $D$, and we obtain an orientable or a nonorientable surface $S_{D, m^{\prime}}$ with $b_{D, m^{\prime}}$ boundary components with even symbols $\circ$ and even symbols $\bullet$. Then, we have

$$
W_{\mathfrak{s p}_{N}}(D)=\sum_{m^{\prime}}(-1)^{s_{m^{\prime}}} n^{b_{D, m^{\prime}}} h^{d},
$$

where $s_{m^{\prime}}$ is the number of $: \chi_{0}^{0}$ and ${ }_{a}^{\circ} \times$ in $S_{D, m^{\prime}}$ and the sum is over all possible admissible edge marking $m^{\prime}$ of $D$. For example, when $D=\left(\begin{array}{l}y_{2}^{y_{1}} \\ y_{3}\end{array}\right), m^{\prime}\left(y_{1}\right)=\stackrel{\circ}{\longrightarrow}, m^{\prime}\left(y_{2}\right)=: \propto\llcorner$, and $m^{\prime}\left(y_{3}\right)=\stackrel{0}{0}$, the surface $S_{D, m^{\prime}}\left(\begin{array}{l}0 \\ 0\end{array}\right.$ boundary components and so contributes $n^{2} h$ to $W_{\mathfrak{s p}_{N}}(\ominus)$. We compute that $W_{\mathfrak{s p}_{N}}(\ominus)=$ $8 n^{3} h+12 n^{2} h+4 n h=2 n(2 n+1)(2 n+2) h=N(N+1)(N+2) h$.

Now let us prove Proposition 9.

Proof of Proposition 9 Let $D$ be a connected trivalent graph. One sees that an admissible edge marking $m^{\prime}: v(D) \rightarrow Y$ in the above description of $W_{\mathfrak{s p}_{N}}$ induces an edge marking $m_{e}: e(D) \rightarrow\{0,1\}$ in the description of $W_{\mathfrak{s o}_{N}}$ in Section 4 , by ignoring the symbols $\circ$ and $\bullet$. Let $m_{e}: e(D) \rightarrow\{0,1\}$ be an edge marking. We construct an admissible marking $m^{\prime}: v(D) \rightarrow Y^{\prime}$ which induces $m_{e}$ as follows. Let $B$ be a boundary component of the surface $S_{D, m_{e}}$. We decompose $B$ into a sequence $\alpha_{1} \beta_{1} \ldots \alpha_{k} \beta_{k}$ of arcs, where $\alpha_{i}$ is one of two arcs in the diagram - or $>$ and $\beta_{i}$ is one of three arcs in the diagram . Let $p_{i}$ be the intersection point of $\beta_{i-1}$ and $\alpha_{i}$ for $1 \leq i \leq k$, where $\beta_{0}:=\beta_{k}$, and $q_{i}$ be the intersection point of $\alpha_{i}$ and $\beta_{i}$ for $1 \leq i \leq k$. Next, we assign $p_{i}$ and $q_{i}$ with $\circ$ or $\bullet$ in such a way that $q_{i-1}$ and $p_{i}$ for $1 \leq i \leq k$ are assigned with the same symbol, where $q_{0}:=q_{k}$, and that if $\alpha_{i}$ is an arc in the diagram — ( resp. $<$ ), then $p_{i}$ and $q_{i}$ are assigned with the same symbol (resp. the different symbol). As the number of $\alpha_{i}$ which is an arc in the diagram $<$ is even and an assignment of $p_{1}$ determines such an assignment, such two assignments exist. A surface $S_{D, m_{e}}$ with any boundary component given one of two possible assignments is said to be decorated. It follows from the definition of $Y^{\prime}$ that a decorated surface $S_{D, m_{e}}$ determines $S_{D, m^{\prime}}$ for an admissible edge marking $m^{\prime}: e(D) \rightarrow Y^{\prime}$ inducing $m_{e}$. For any edge marking $m_{e}$, there exist $2^{b_{D}, m_{e}}$ admissible edge markings $m^{\prime}$ that induces $m_{e}$. Moreover, it holds that for any admissible edge marking $m^{\prime}$, there exists an edge marking $m_{e}$ such that a decorated surface $S_{D, m_{e}}$ coincides with $S_{D, m^{\prime}}$. Noting that the number of $\_$, @ o on each boundary component of $S_{D, m^{\prime}}$ is even, one also sees that if admissible edge markings $m_{1}^{\prime}$ and $m_{2}^{\prime}$ induce the same edge marking $m_{e}$, then $s_{m_{1}^{\prime}} \equiv s_{m_{2}^{\prime}}(\bmod 2)$. Consequently, we obtain that

$$
W_{\mathfrak{s p}_{N}}(D)=\sum_{m_{e}}(-1)^{s_{m^{\prime}}} 2^{b_{D, m_{e}}} n^{b_{D, m_{e}}} h^{d},
$$

where the sum is over all possible edge marking $m_{e}: e(D) \rightarrow\{0,1\}, m^{\prime}$ is an admissible edge marking inducing $m_{e}$, and $d$ is the degree of $D$. Moreover, by the definition of $s_{m_{e}}$ and $s_{m^{\prime}}$, we have that $s_{m_{e}}=s_{m^{\prime}}+j_{m^{\prime}}$, where $j_{m^{\prime}}$ is the number of $: \chi_{\bullet}, \mathcal{L}_{0}$ in $S_{D, m^{\prime}}$. Hence, we obtain that

$$
W_{\mathfrak{s p}_{N}}(D)=\sum_{m_{e}}(-1)^{s_{m_{e}}-j_{m^{\prime}}}(2 n)^{b_{D, m_{e}}} h^{d}=\sum_{m_{e}}(-1)^{s_{m_{e}}-j_{m^{\prime}}} N^{b_{D, m_{e}}} h^{d} .
$$


From the formula (10), to prove Proposition 9, it is enough to show that $d+b_{D, m_{e}} \equiv j_{m^{\prime}}$ $(\bmod 2)$. We remark that $2-g_{D, m^{\prime}}^{\prime}=-d+b_{D, m_{e}}$. In the case that $j_{m^{\prime}}=0$, one sees that $S_{D, m^{\prime}}$ is an orientable surface and that $-d+b_{D, m_{e}}=2-2 g\left(S_{D, m^{\prime}}\right) \equiv 0=j_{m^{\prime}}(\bmod 2)$. Suppose that $j_{m^{\prime}} \neq 0$. From the definition of an admissible edge marking, we see that the surface $S_{D, m^{\prime}}$

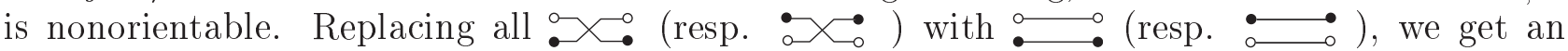
orientable surface $S_{D, m^{\prime}}^{o}$. Then, it follows that $g_{D, m^{\prime}}^{\prime} \equiv 2 g\left(S_{D, m^{\prime}}^{o}\right)+j_{m^{\prime}} \equiv j_{m^{\prime}}(\bmod 2)$ and so we get that $-d+b_{D, m_{e}}=2-g_{D, m^{\prime}}^{\prime} \equiv j_{m^{\prime}}(\bmod 2)$. This completes the proof of Proposition 9 .

Remark. Proposition 9 is noted as Exercise 6.37 in [2]. It can also be obtained from a result on the weight system associated with the super Lie algebra $\mathfrak{o s p}(m, n)$ in [9], noting that $\mathfrak{o} \mathfrak{s p}(m, 1)=\mathfrak{s o}_{m}$ and that $\mathfrak{o} \mathfrak{s p}(1, n)=\mathfrak{s p}_{n}$.

\section{REFERENCES}

[1] D. Bar-Natan, Weights of Feynman diagrams and the Vassiliev knot invariants, preprint, available at

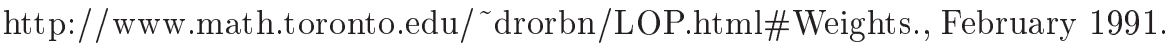

[2] _ On the Vassiliev knot invariant, Topology 34 (1995), 423-472.

[3] P. Cvitanovic, Group Theory. Birdtracks, Lie's, and exceptional groups, Princeton University Press, Princeton, 2008.

[4] S. Garoufalidis, T.T.Q. Le, and M. Mariño, Analycity of the free energy of a closed 3-manifold, SIGMA 4 (2008), no. 080, 20 pages.

[5] S. Garoufalidis and M. Mariño, Universality and asymptotics of graph counting problems in unoriented surfases, to appear in Journal of Combinatorial Theory A, math.CO/0812.1295.

[6] T. T. Q. Le, J. Murakami, and T. Ohtsuki, On a universal perturbative invariant of 3-manifolds, Topology 37 (1998), no. 3, 539-574.

[7] T. Ohtsuki, Quantum invariants. A study of knots, 3-manifolds, their sets, Series on Knots and Everything, vol. 29, World Scientific Publishing Co., Inc., River Edge, NJ, 2002.

[8] S. Sinha and C. Vafa, SO and Sp Chern Simons at large N, hep-th/0012136.

[9] P. Vogel, Algebraic structures on modules of diagrams, available at http://www.math.jussieu.fr/ ${ }^{\sim}$ vogel/.

Dedicated to Professor Akio Kawauchi on the occasion of his 60th birthday

Faculty of Mathematics, Kyushu University, Fukuoka, JAPAN, TTAkata@math.KyUshu-U.AC.JP 